\title{
A 40-month multicentre, randomised placebo- controlled study to assess the efficacy and carry- over effect of repeated intra-articular injections of hyaluronic acid in knee osteoarthritis: the AMELIA project
}

\author{
F Navarro-Sarabia, ${ }^{1}$ P Coronel, ${ }^{2}$ E Collantes, ${ }^{3} \mathrm{~F} J$ Navarro, ${ }^{4}$ A Rodriguez de la Serna, ${ }^{5}$ \\ A Naranjo, ${ }^{6} \mathrm{M}$ Gimeno, ${ }^{7} \mathrm{G}$ Herrero-Beaumont, ${ }^{8}$ on behalf of the AMELIA study group
}

${ }^{1}$ Rheumatology Department, Hospital Universitario Virgen Macarena, Sevilla, Spain ${ }^{2}$ Scientific Department, Tedec Meiji Farma, Alcalá de Henares, Madrid, Spain

${ }^{3}$ Rheumatology Department, Hospital Reina Sofía, Córdoba, Spain

4Rheumatology Department, Hospital General de Elche,

Alicante, Spain

${ }^{5}$ Rheumatology Department, Hospital Santa Creu i Sant Pau, Barcelona, Spain

${ }^{6}$ Rheumatology Department, Hospital de Gran Canaria

Dr Negrín, Las Palmas de Gran Canaria, Spain

${ }^{7}$ R\&D Department, Tedec Meiji Farma, Alcalá de Henares, Madrid, Spain

${ }^{8}$ Rheumatology Department, Fundación Jiménez Díaz.

Madrid, Spain

\section{Correspondence to}

F Navarro-Sarabia,

Rheumatology Department, Hospital Universitario Virgen

Macarena, Avda Doctor

Fedriani 3, 41011 Sevilla, Spain;

federico.navarro.sspa@

juntadeandalucia.es

Accepted 13 June 2011

Published Online First

17 August 2011

\begin{abstract}
Objective AMELIA (OsteoArthritis Modifying Effects of Long-term Intra-articular Adant) was designed to compare against placebo the efficacy and safety of repeated injections of hyaluronic acid (HA) and its effect on disease progression over 40 months.

Methods A multicentre, randomised, patient and evaluator-blinded, controlled study in 306 patients fulfilling American College of Rheumatology criteria for knee osteoarthritis, radiological grades II-III (KellgrenLawrence) and joint space width $\geq 2 \mathrm{~mm}$. Patients received four cycles of five intra-articular HA or placebo injections with a follow-up of 6 months after the first and second cycles, and 1 year after the third and fourth cycles. Osteoarthritis Research Society International (OARSI) 2004 responder criteria were used to assess efficacy. The consumption of rescue medication was a secondary outcome. Adverse events were recorded for safety purposes.
\end{abstract}

Results At the 40-month visit significantly more patients responded to HA compared with placebo (OARSI 2004, $p=0.004)$. The number of responders to HA increased through the study, whereas those to placebo did not change. Significant differences were also found in favour of HA for each individual component of the OARSI 2004. No safety problems were recorded.

Conclusions The results of AMELIA offer pioneer evidence that repeated cycles of intra-articular injections of HA not only improve knee osteoarthritis symptoms during the in-between cycle period but also exert a marked carry-over effect for at least 1 year after the last cycle. In this respect, it is not possible to establish if this carry-over effect reflects true osteoarthritis remission or just a modification of the disease's natural course. ClinicalTrials.gov number, NCT00669032

Osteoarthritis is a chronic disorder characterised by joint cartilage degeneration as the central feature associated with concomitant changes in synovium and subchondral bone metabolism. ${ }^{1}$ Osteoarthritis of the knee, the principal large joint to be affected, results in disabling symptoms in $10 \%$ of people older than 55 years, a quarter of whom are severely affected. ${ }^{2}$ Most current pharmacological options are limited to alleviating pain and improving functional activity but effective therapeutic alternatives to slow disease progression are also needed.
Hyaluronic acid (HA) is a key molecule in joint biomechanics. In osteoarthritis and other arthropathies, the reduction in concentration and molecular weight of endogenous HA greatly alters the properties of the synovial fluid, causing cartilage damage and worsening osteoarthritis symptoms. ${ }^{3}$ Treatment with exogenous HA contributes to restoring the elastic and viscous properties of the synovial fluid, resulting in pain reduction and functional improvement. Besides this mechanical action, different studies have confirmed that HA interacts with mediators of inflammation and matrix turnover in joint cells, reduces the apoptosis of chondrocytes and exerts a biosynthetic chondroprotective effect. ${ }^{4-9}$

This dual mechanism of action may account for the particular effect of HA on clinical symptoms of osteoarthritis. Unlike traditional analgesics and nonsteroidal anti-inflammatory drugs (NSAID), HA is not a rapidly acting agent, but its clinical efficacy on pain and function shows a carry-over effect that extends the results for a long time after the administration is stopped, identifying it as a symptomatic slow-acting class compound (SYSADOA). ${ }^{10} 11$

Although the use of intra-articular HA injections for the relief of pain in people with knee osteoarthritis is recommended by scientific societies, ${ }^{12} 13$ the characteristics of the patients who are most likely to benefit from this treatment need to be defined appropriately, and the role of HA in disease progression is still under discussion. ${ }^{12-15}$ Unfortunately, the lack of long-term studies contributes to this uncertainty. In addition, the variety of HA have different physicochemical characteristics and thus cannot be expected to have the same clinical effect. ${ }^{4} 1617$

The current trial AMELIA (OsteoArthritis Modifying Effects of Long-term Intra-articular Adant) was conducted to compare the efficacy and safety of repeated injections of HA compared with placebo over a period of 40 months.

\section{METHODS}

\section{Study design}

This was a randomised, patient and evaluator blinded, placebo-controlled study with parallel groups. Patients with osteoarthritis of the knee were randomly assigned to receive intra-articular 
injections of $2.5 \mathrm{ml} 1 \%$ sodium hyaluronate with a mean molecular weight of 900000 daltons, obtained through a fermentation process from strains of Streptococcus zoopidemicus (Adant; Tedec-Meiji Farma, Madrid, Spain) or placebo injections $(2.5 \mathrm{ml}$ of saline solution). The study consisted of four treatment cycles of five weekly injections each one. The follow-up periods were 6 months long after the first and second cycles and 1 year long after the third and fourth cycles, resulting in a total study duration of 40 months (figure 1). The repeated cycles were administered regardless of whether the patients had symptoms or not.

Both treatments were packaged identically in order to maintain the blinding conditions. A computer-generated randomised list was used to provide balanced blocks of four patients each. Allocation to treatment as well as efficacy and safety evaluation was performed by a blinded physician, while an unblinded physician was responsible for treatment administration. The injections were non-ultrasound guided and three approaches were permitted: medial/lateral (extended knee) and infrapatellar (flexed knee).

At the screening visit the patients were assessed by the blinded physician for fulfilment of the entry criteria, demographic characteristics and medical history. Knee radiographs were also obtained. Eligible patients were randomly assigned 1:1 to receive intra-articular injections of HA or placebo. In the case of bilateral affectation only the more symptomatic knee at baseline was considered for the study, although the other knee could be treated with the same assigned treatment. Patients with effusion had the joint aspirated before the administration of treatment. Concomitant medications for chronic pathologies other than osteoarthritis were also recorded. Acetylsalicylic acid (maximum $300 \mathrm{mg} /$ day) for vascular prevention, paracetamol up to $4 \mathrm{~g}$ /day as rescue medication as well as short cycles of NSAID were permitted. However, for $24 \mathrm{~h}$ and 1 week before efficacy evaluation, patients were required to abstain from any paracetamol or NSAID, respectively. During the whole study period corticosteroid injections were not permitted in the target knee. Only two injections were allowed in the contralateral knee if necessary and no more than two injections per year in any other joint than the knee.

\section{Patients' inclusion and exclusion criteria}

Eligible patients were men and women of at least 45 years of age with knee osteoarthritis in the medial tibiofemoral compartment according to the American College of Rheumatology ${ }^{18}$ with grade II to III radiographic stage osteoarthritis ${ }^{19}$ and minimum medial femorotibial joint space width of the target knee of $2 \mathrm{~mm}$ or greater. Patients were required to have pain of $55 \mathrm{~mm}$ or greater on a visual analogue scale (VAS) at any time during the week before inclusion.
Main exclusion criteria were body mass index greater than $32 \mathrm{~kg} / \mathrm{m}^{2}$, a history of trauma or surgery in the target knee, arthroscopy surgery during the year before inclusion, joint inflammatory diseases and/or microcrystalline arthropathies, coagulation/platelet disorders or any concomitant disease that could interfere with the evaluation. The administration of intraarticular steroids in the previous 3 months, HA injections during the past year or NSAID treatment during 2 weeks before inclusion were also reasons for exclusion.

\section{Evaluation of efficacy}

The primary efficacy outcome was the percentage of subjects with a clinical response according to Osteoarthritis Research Society International (OARSI) 2004 criteria $^{20}$ at the end of follow-up. Patients were classified as responders if the pain or physical function score decreased at least $50 \%$ and at least $20 \mathrm{~mm}$ on the VAS, or if two of the following three findings were recorded: a decrease in pain of at least $20 \%$ or at least 10 $\mathrm{mm}$ on the VAS, a decrease in physical function of at least $20 \%$ and at least $10 \mathrm{~mm}$ on the VAS, or an increase in the score of the patient's global assessment by at least $20 \%$ and at least $10 \mathrm{~mm}$ on the VAS.

Secondary outcomes included the percentage of subjects with clinical response according to OMERACT-OARSI criteria at each follow-up visit; each component of OMERACT-OARSI (reduction in pain, improvement in function using the Western Ontario and McMaster Universities Osteoarthritis Index function subscale and in patients' global assessment (all of them measured using VAS) and consumption of rescue medication for osteoarthritis (paracetamol and NSAID) throughout the study).

\section{Evaluation of safety}

Treatment safety and tolerability was evaluated based on the incidence and type of adverse events (with special attention to allergic reactions such as skin rash, urticaria, pruritus, swelling and/or erythema) and the results of blood laboratory tests and physical examinations throughout the duration of the study. Safety analyses were performed in those patients who received at least one intra-articular injection (safety population).

\section{Statistical methods}

The study was designed to have a statistical power of $80 \%$ to detect a difference of at least $20 \%$ of patients responding to treatment compared with placebo (expected response rate up to $30 \%$ ), with a two-sided significance level of less than $5 \%$. Given the specified statistical power and assuming up to a $40 \%$ dropout rate, the study was planned to include a total of 300 patients (150 per treatment arm).

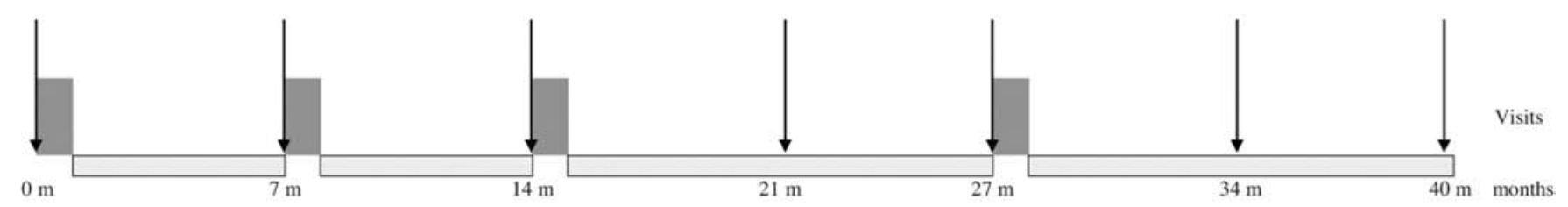

5 i.a. injections HA/Placebo

Figure 1 Study diagram. HA, hyaluronic acid; i.a., intra-articular. 
For quantitative variables, mean, median, SD, maximum and minimum values were calculated. Student's t test was used to compare independent variables following a normal distribution and the Mann-Whitney $U$ test was used if the applicability conditions were not present. Qualitative variables were expressed as total number and relative frequencies. Pearson's $\chi^{2}$ test was used for comparisons of frequencies between groups.

The main study population included all randomly assigned patients with at least one efficacy assessment after randomisation (the modified intention-to-treat population). A sensitivity analysis was performed to assess the imputation method for handling missing data using the mixed method of repeated measures and the last observation carried forward. The last observation carried forward proved to be the more conservative method and was then applied. All statistical tests were performed using SAS software version 9.2.

\section{RESULTS}

\section{Disposition of patients and demographic characteristics}

Recruiting started in October 2003 and the last follow-up was performed on July 2009. The 19 participating centres screened a total of 446 patients, of whom 140 (31.4\%) were screening failures. Five patients did not provide any efficacy data after randomisation and were not included in the analysis of efficacy, leaving a total of 301 patients in the intention-to-treat population. A total of 109 and 94 patients receiving HA or placebo, respectively, completed the study (figure 2 ).

The clinical and demographic characteristics of the patients did not differ at baseline (table 1). Overall, the great majority of the patients were women (83.7\%) the mean age was 63.4 years, with a body mass index of 28.6. The mean duration of knee osteoarthritis was 7.5 years and a mean joint space width value of $3.5 \mathrm{~mm}$. A total of $77.85 \%$ of HA patients and $82.24 \%$ in the placebo group had bilateral osteoarthritis $(\mathrm{p}=0.341)$. Of them, $55.17 \%$ of HA patients and $56.02 \%$ of the placebo group ( $\mathrm{p}=0.7992)$ were also treated in the contralateral knee. No differences between groups were found related to the administration approach.

\section{Treatment efficacy}

At the end of follow-up (40 months) significantly more patients receiving $\mathrm{HA}$ responded to treatment in comparison with

Table 1 Demographic and baseline clinical characteristics of study groups

\begin{tabular}{lll}
\hline & HA (n=153) & Placebo (n=153) \\
\hline Women, n (\%) & $128(83.7)$ & $128(83.7)$ \\
Age, years (mean (SD)) & $63(8.2)$ & $63.9(8.9)$ \\
BMI, kg/m² (mean (SD)) & $28.4(2.7)$ & $28.7(2.6)$ \\
Duration knee osteoarthritis, years (mean (SD)) & $6.9(6.8)$ & $8.1(8.4)$ \\
Osteoarthritis baseline characteristics & & \\
$\quad$ Pain, VAS 0-100 (mean (SD)) & $69.7(11.1)$ & $71.2(11.2)$ \\
$\quad$ Morning stiffness, <30 min (n (\%)) & $142(92.8)$ & $133(86.9)$ \\
Joint crackles, n (\%) & $141(92.2)$ & $138(90.2)$ \\
Kellgren-Lawrence grade, n (\%) & & \\
II & $108(70.6)$ & $114(74.5)$ \\
III & $45(29.4)$ & $39(25.5)$ \\
Joint space width, mm (mean (SD)) & $3.5(0.82)$ & $3.5(0.89)$ \\
WOMAC, VAS 0-100 (mean (SD)) & & \\
Total & $55.7(15.5)$ & $58.2(14.7)$ \\
Pain & $56.0(16.7)$ & $56.7(15.2)$ \\
Stiffness & $54.8(23.0)$ & $54.8(22.4)$ \\
Function & $57.1(17.0)$ & $59.1(15.1)$
\end{tabular}

BMI, body mass index; HA, hyaluronic acid; VAS, visual analogue scale; WOMAC, Western Ontario and McMaster Universities Osteoarthritis Index.

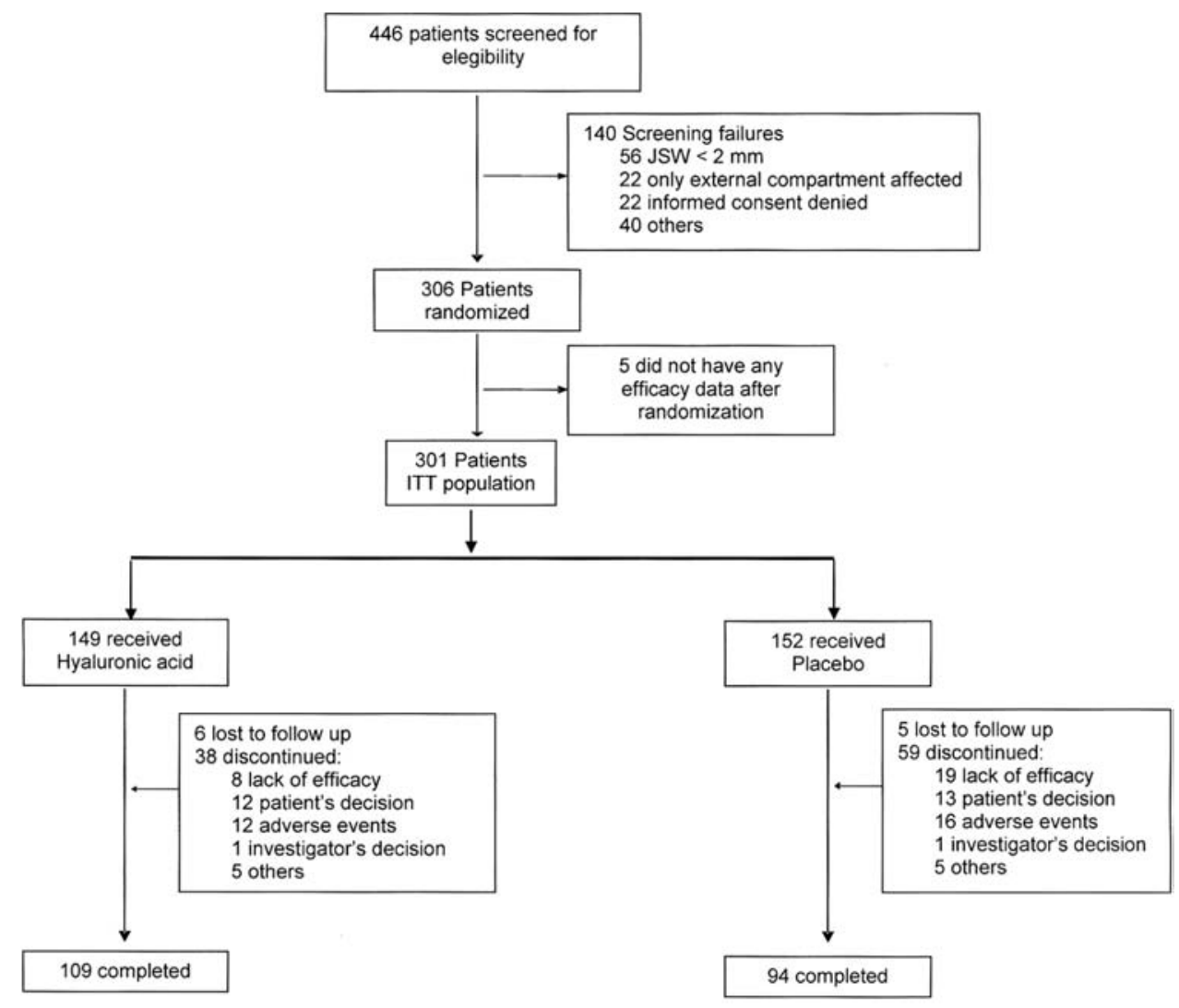

Figure 2 Patients' disposition for clinical assessment. JSW, joint space width. 


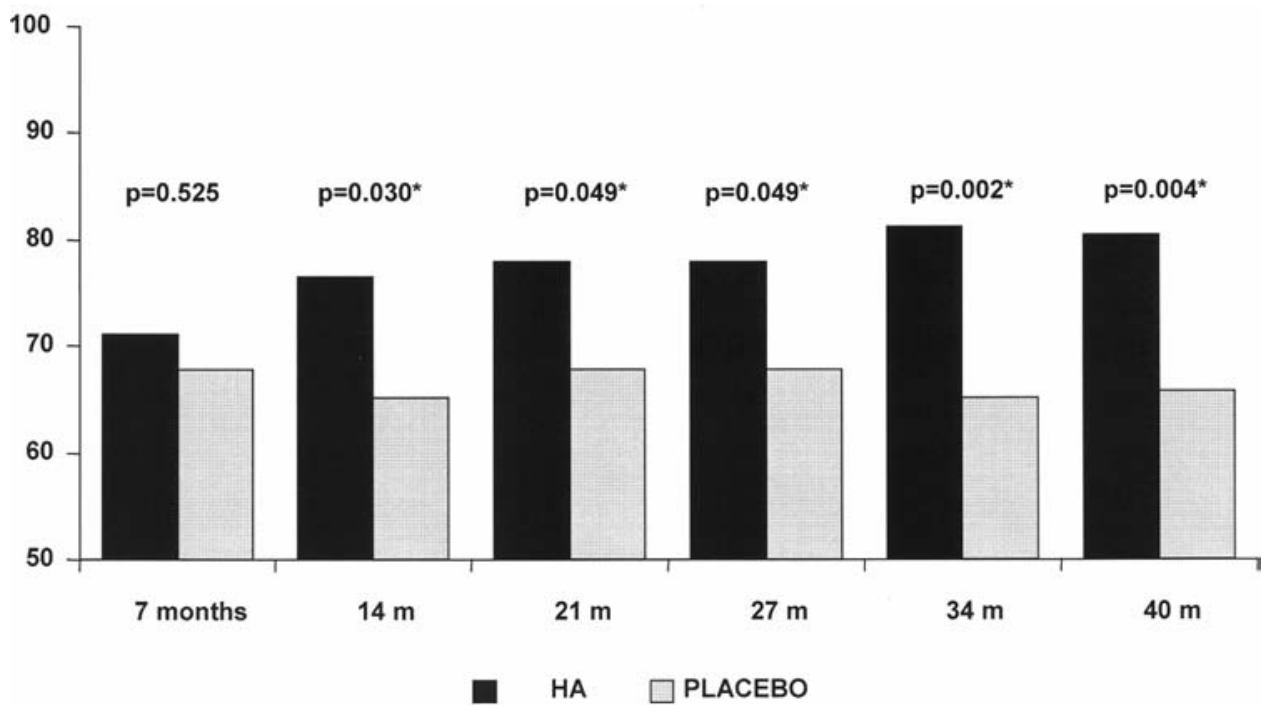

Figure 3 Evolution of responders Osteoarthritis Research Society International, 2004. HA, hyaluronic acid.

Table 2 Summary of results from primary and secondary outcomes assessment (intention-to-treat population)

\begin{tabular}{|c|c|c|c|}
\hline & $\begin{array}{l}\text { HA } \\
(n=149)\end{array}$ & $\begin{array}{l}\text { Placebo } \\
(n=152)\end{array}$ & p Value \\
\hline \multicolumn{4}{|l|}{ Primary outcome } \\
\hline $\begin{array}{l}\text { Responders OARSI } 2004 \\
\text { at end of follow-up, n (\%) }\end{array}$ & $120(80.5)$ & $100(65.8)$ & 0.004 \\
\hline \multicolumn{4}{|l|}{ Secondary outcomes } \\
\hline \multicolumn{4}{|c|}{ Responders OARSI 2004 at each cycle assessments, n (\%) } \\
\hline 7 months & $106(71.1)$ & $103(67.8)$ & 0.525 \\
\hline 14 months & $114(76.5)$ & $99(65.1)$ & 0.030 \\
\hline 21 months & $116(77.9)$ & $103(67.8)$ & 0.049 \\
\hline 27 months & $116(77.9)$ & $103(67.8)$ & 0.049 \\
\hline 34 months & $121(81.2)$ & $99(65.1)$ & 0.002 \\
\hline $\begin{array}{l}\text { Pain or function reduction } 50 \% \\
(20 \mathrm{~mm}), \mathrm{n}(\%)\end{array}$ & $97(65.1)$ & $79(52.0)$ & 0.021 \\
\hline $\begin{array}{l}\text { Overall pain reduction } 20 \% \\
(10 \mathrm{~mm}), \mathrm{n}(\%)\end{array}$ & $118(79.2)$ & $103(67.8)$ & 0.025 \\
\hline $\begin{array}{l}\text { Function improvement 20\% } \\
(10 \mathrm{~mm}), \mathrm{n}(\%)\end{array}$ & $105(70.5)$ & $88(57.9)$ & 0.023 \\
\hline $\begin{array}{l}\text { Patient's global assessment } \\
\text { reduction } 20 \%(10 \mathrm{~mm}), \mathrm{n}(\%)\end{array}$ & $111(74.5)$ & $88(57.9)$ & 0.002 \\
\hline $\begin{array}{l}\text { Mean consumption of } \\
\text { paracetamol, mg/day (SD) }\end{array}$ & $408.8(644.2)$ & $451.4(925.8)$ & NS \\
\hline
\end{tabular}

HA, hyaluronic acid; OARSI, Osteoarthritis Research Society International.

placebo according to OARSI 2004 criteria $(\mathrm{p}=0.004)$, the number of responders being $22 \%$ higher in HA group after the four treatment cycles (RR 1.22, 95\% CI 1.07 to 1.41 ).

The number of responders to HA injections progressively increased after each treatment cycle (from $71.1 \%$ to $80.5 \%$ ), whereas responses to placebo remained fairly stable (from $67.8 \%$ to $65.8 \%)$.

This progression gave results with strong statistical significance and differences between the two groups from the second until the last evaluation at 40 months (figure 3). Among those non-responders after the first cycle, up to $54 \%$ of $\mathrm{HA}$ and $38 \%$ of placebo patients evolved positively over the study. At the 40-month visit the number of responders in this subgroup was $54 \%$ with HA versus $31 \%$ in the placebo group, $(\mathrm{p}=0.026)$.

All of the OARSI components (pain, function and patient global assessment) were analysed at the end of the study, showing that the degree of improvement in the HA group was significantly higher compared with placebo ( $p$ values $0.025,0.023$ and 0.002 , respectively) (table 2 ).

A total of $26.8 \%$ of patients receiving HA did not complete the study compared with $38.2 \%$ in the placebo group. It is noteworthy that the number of losses due to lack of efficacy were significantly higher in the placebo group $(p=0.027)$. The demographic and baseline characteristics of completers and dropouts were analysed, and no differences were found with the exception of age in the placebo group, with the completers being younger than the dropouts $(p=0.047)$. Aspiration in the target knee was performed in $22.82 \%$ of patients in the HA group and $21.05 \%$ of the placebo group $(p=0.712)$, with a median of two aspirations per patient in both groups during the overall study period.

Overall, rescue medication (paracetamol/NSAID) was consumed during the study by $71.1 \%$ and $71.7 \%$ of the HA and placebo patients, respectively. Paracetamol was consumed by $48 \%$ of the patients and the mean daily dose during the study experienced a $27 \%$ reduction in the HA group compared with baseline versus only a $4 \%$ reduction in the placebo group (table 2 ). A logistic regression analysis was performed with no differences between the HA and placebo ( $p=0.9129)$ groups, concluding that rescue medication did not interfere with the clinical assessment of patients.

\section{Safety}

The number of patients who experienced at least one adverse event was the same in both treatment groups, with an overall frequency of $83.0 \%$. Twenty-two patients (11 in each group) experienced a total of 29 related adverse events. Most of them were related to the study intervention, such as local bleeding, pain of mild intensity or allergic reaction, none of them was serious. The frequency and types of related adverse events are summarised in table 3. During the overall study period, there were no abnormalities in vital signs, clinical findings or laboratory parameters that could be considered as being related to the treatment. At the end of the study, 513 and 487 cycles were administered in the HA and placebo groups, respectively, providing a rate of 0.029 related adverse events per cycle in both groups. 
Table 3 Related adverse events

\begin{tabular}{|c|c|c|c|c|}
\hline & & & HA $(n=153)$ & Placebo $(n=153)$ \\
\hline $\begin{array}{l}\text { Related adverse events } \\
\text { (n (\%)) }\end{array}$ & & & $15(9.8)$ & $14(9.1)$ \\
\hline Mild & & & $7(4.6)$ & $12(7.8)$ \\
\hline \multirow[t]{3}{*}{ Moderate } & & & $8(5.2)$ & $2(1.3)$ \\
\hline & $H A(n=153)$ & & Placebo $(n=153)$ & \\
\hline & Mild & Moderate & Mild & Moderate \\
\hline Allergic reaction & $\begin{array}{l}2(1.3) \\
(1 \text { rash, } 1 \text { swelling })\end{array}$ & $\begin{array}{l}1(0.7) \\
(1 \text { rash) }\end{array}$ & $\begin{array}{l}3(1.9) \\
\text { (3 rash) }\end{array}$ & 0 \\
\hline Pain at injection site & $2(1.3)$ & $4(2.6)$ & $2(1.3)$ & 0 \\
\hline Bleeding at injection site & $2(1.3)$ & 0 & $6(3.9)$ & 0 \\
\hline Arthralgia & 0 & $2(1.3)$ & $1(0.7)$ & $1(0.7)$ \\
\hline Others & $1(0.7)$ & $1(0.7)$ & 0 & $1(0.7)$ \\
\hline
\end{tabular}

\section{DISCUSSION}

This study has assessed the efficacy and safety of repeated intraarticular injections of HA in knee osteoarthritis patients, showing a symptomatic relief of pain and an improvement in function and patient global assessment during a follow-up period of 40 months. At the end of the study, the percentage of responders according to OARSI 2004 criteria was $22 \%$ higher in the HA group than in the placebo group ( $\mathrm{p}=0.004)$.

In recent years, consistent systematic reviews and meta-analyses $^{1021-26}$ have concluded that HA is superior to placebo in controlling knee osteoarthritis symptoms. ${ }^{24-26}$ Although a number of controlled studies have shown that this treatment is comparable in efficacy to systemic forms of active intervention such as steroids or NSAID, ${ }^{22} 23$ some other studies have questioned the efficacy of the treatment with HA. ${ }^{21} 27$ The study design weaknesses and the enormous placebo effect in osteoarthritis clinical trials as well as the specific characteristics of the HA used can account for these contradictory results. There are two crucial aspects that have to be strongly considered in the design of clinical trials aimed to demonstrate a SYSADOA effect in osteoarthritis: the time when the analgesia starts and the duration of the carry-over effect. Furthermore, the various HA derivatives that are available on the market are synthesised using different methods and formulated in different physical forms, and thus a uniform efficacy and safety profile should not be expected. ${ }^{4} 161728$

The starting time of the analgesic effect could not be well captured in some short-term clinical trials, and our study was not designed to address it. In fact, despite some patients going into remission at the first assessment performed 7 months after the first cycle, this effect could not be demonstrated, probably masked by the high efficacy rates found in the placebo group at that time. However, the efficacy of placebo remained quite stable throughout the study period, whereas the responder rate in the HA group increased, the differences being truly significant from 1 year onwards $(p<0.05)$. Several randomised controlled trials in osteoarthritis failed to demonstrate the superiority of active treatment over placebo, 27 29-32 reaching efficacy rates up to $60 \%$ even with orally administered placebos, ${ }^{30}$ making it difficult for the other treatments to surpass this level. ${ }^{10}$ Moreover, the efficacy of placebo has been confirmed in osteoarthritis interventions, and increases with the expectations of the patient with respect to the treatment and the use of invasive administration routes. ${ }^{32}$ In AMELIA, however, the success of the study was in fact accentuated by the high placebo efficacy detected, making the results found $(80.5 \%$ of responders in the HA group at the end of follow-up compared with $65.8 \%$ for placebo patients), even clinically meaningful and remarkable. ${ }^{30}$ The RR used to quantify the effect size established a probability of success of HA of $22 \%$ higher than placebo 1 year after the last cycle was administered.

The AMELIA design enables us to gain a better understanding of the clinical response of symptomatic knee osteoarthritis patients subject to HA injections by looking for the carry-over analgesic effect duration. AMELIA was able to detect symptomatic effects even 1 year after the last HA administration cycle, giving us unprecedented information about the HA therapeutic profile. These results are in line with those reported previously, granting HA greater efficacy than $\mathrm{NSAID}^{23}$ and than steroids after 5-8 weeks post-treatment, ${ }^{22} 33$ even though previous studies do not provide long-term data. We cannot ascertain whether the pre-established therapeutic schedule, consisting of administering HA even to patients without symptoms, could contribute to the carry-over effect, but it could be a plausible explanation. In this regard, it is not possible to establish whether this carryover effect reflects disease remission or just a modification of the natural course of the disease.

Overall, the use of rescue medication during the study was low. Although the mean paracetamol consumption was $23 \%$ lower in the HA group, the differences were not statistically significant, probably due to the high data dispersion. Certainly, the way of controlling rescue medication use is a study limitation because patients were asked at every assessment visit about the frequency and doses of consumption, but it was not delivered in hand, leading to insufficient recording and an inherent inaccuracy. In any case, however, the logistic regression analysis concluded that the use of rescue medication did not interfere with the assessment of the clinical outcome.

Adverse reactions were scarce and were related to the administration procedure. All of them were short lasting and of mild or moderate intensity.

The results of AMELIA reveal that repeated cycles of intraarticular injections of HA not only improve knee osteoarthritis symptoms during the in-between cycle period, but also exert a marked carry-over effect for at least 1 year after the last injections. In this regard, it is not possible to establish whether this carry-over effect reflects a true disease remission or just a modification of the natural course of the disease.

AMELIA Study Group J Toyos, Rheumatology Department, H Virgen Macarena, Sevilla; B Hernández-Cruz, Rheumatology Department, H Virgen Macarena, Sevilla; J Belzunegui, Rheumatology Department, Hospital Ntra Sra de Aránzazu, San Sebastián; S García, Rheumatology, Department, Hospital Puerta del Mar, Cádiz; M E Brito, Rheumatology Department, Hospital Ramón y Cajal, Madrid; J C Acebes, Rheumatology Department, Fundación Jiménez Díaz, Madrid; J L Guerra, 
Rheumatology Department, Hospital Arquitecto Marcide, El Ferrol (La Coruña): J Pujol, Rheumatology Department, Hospital Sant Pau i Santa Tecla, Tarragona; F Blanco, Rheumatology Department, Hospital Juan Canalejo, La Coruña; P Benito, Rheumatology Department, Hospital del Mar, Barcelona; E Chamizo, Rheumatology Department, Hospital de Mérida, Mérida (Badajoz); F A Martínez, Rheumatology Department, Hospital Virgen de la Arrixaca, Murcia; R García, Rheumatology Department, Hospital Clínico Universitario Virgen de la Victoria, Málaga:

M Guzmán, Rheumatology Department, Hospital Virgen de las Nieves, Granada; M García, Traumatology Department, Hospital Vall D’Hebrón, Barcelona; J Mulero, Rheumatology Department, Hospital Puerta de Hierro, Madrid.

Acknowledgements The authors would like to thank J Suarez for assisting them with the English translation. They also thank JJ García and M Amor (PIVOTAL SL) for the statistical analysis of the results.

Funding This study was supported by Tedec Meiji Farma SA

Competing interests $\mathrm{PC}$ and MG work at Tedec Meiji Farma SA. The other authors received research funds from Tedec Meiji Farma SA as study investigators

Patient consent Obtained

Ethics approval The study was performed in accordance with the principles of good clinical practice guidelines and in compliance with the declaration of Helsinki. The study was conducted with the approval of the ethics committees from each of the centres where the study was performed.

Provenance and peer review Not commissioned; externally peer reviewed.

\section{REFERENCES}

1. Herrero-Beaumont G, Roman-Blas JA, Castañeda S, et al. Primary osteoarthritis no longer primary: three subsets with distinct etiological, clinical, and therapeutic characteristics. Semin Arthritis Rheum 2009;39:71-80.

2. Peat G, McCarney R, Croft P. Knee pain and osteoarthritis in older adults: a review of community burden and current use of primary health care. Ann Rheum Dis 2001;60:91-7.

3. Fam H, Bryant JT, Kontopoulou M. Rheological properties of synovial fluids. Biorheology 2007:44:59-74.

4. Ghosh P, Guidolin D. Potential mechanism of action of intra-articular hyaluronan therapy in osteoarthritis: are the effects molecular weight dependent? Semin Arthritis Rheum 2002;32:10-37.

5. Greenberg DD, Stoker A, Kane S, et al. Biochemical effects of two different hyaluronic acid products in a co-culture model of osteoarthritis. Osteoarthritis Cartilage 2006;14:814-22

6. Karna E, Miltyk W, Palka JA, et al. Hyaluronic acid counteracts interleukin-1-induced inhibition of collagen biosynthesis in cultured human chondrocytes. Pharmacol Res 2006:54:275-81.

7. Takahashi K, Goomer RS, Harwood F, et al. The effects of hyaluronan on matrix metalloproteinase-3 (MMP-3), interleukin-1 beta (IL-1beta), and tissue inhibitor of metalloproteinase-1 (TIMP-1) gene expression during the development of osteoarthritis. Osteoarthritis Cartilage 1999;7:182-90.

8. Takahashi K, Hashimoto S, Kubo T, et al. Effect of hyaluronan on chondrocyte apoptosis and nitric oxide production in experimentally induced osteoarthritis. J Rheumatol 2000:27:1713-20.

9. Waddell DD, Kolomytkin OV, Dunn S, et al. Hyaluronan suppresses IL-1betainduced metalloproteinase activity from synovial tissue. Clin Orthop Relat Res 2007:465:241-8

10. Bruyère $\mathbf{0}$, Burlet $\mathrm{N}$, Delmas $\mathrm{PD}$, et al. Evaluation of symptomatic slow-acting drugs in osteoarthritis using the GRADE system. BMC Musculoskelet Disord 2008;9:165.

11. Dougados M. Symptomatic slow-acting drugs for osteoarthritis: what are the facts? Joint Bone Spine 2006;73:606-9.

12. Altman RD, Hochberg MC, Moskowitz RW, et al. Recommendations for the medical management of osteoarthritis of the hip and knee. 2000 update. American College of Rheumatology Subcommittee on Osteoarthritis Guidelines. Arthritis Rheum 2000;43:1905-15.

13. Jordan KM, Arden NK, Doherty M, et al. EULAR Recommendations 2003: an evidence based approach to the management of knee osteoarthritis: report of a Task Force of the Standing Committee for International Clinical Studies Including Therapeutic Trials (ESCISIT). Ann Rheum Dis 2003;62:1145-55.

14. The National Collaborating Centre for Chronic Conditions. Osteoarthritis: National Clinical Guideline for Care and Management in Adults. London: Royal College of Physicians, 2008.

15. Zhang W, Moskowitz RW, Nuki G, et al. OARSI recommendations for the management of hip and knee osteoarthritis, Part II: OARSI evidence-based, expert consensus guidelines. Osteoarthritis Cartilage 2008;16:137-62.

16. Prieto JG, Pulido MM, Zapico J, et al. Comparative study of hyaluronic derivatives: rheological behaviour, mechanical and chemical degradation. Int J Biol Macromol 2005;35:63-9.

17. Sánchez-Lázaro J, Díaz-Gállego L. The clinical impact of physical-chemical characteristics of different hyaluronic acids as a treatment for osteoarthritis. Int J Biol Macromol 2006;38:300-1.

18. Altman R, Asch E, Bloch D, et al. Development of criteria for the classification and reporting of osteoarthritis. Classification of osteoarthritis of the knee. Diagnostic and Therapeutic Criteria Committee of the American Rheumatism Association. Arthritis Rheum 1986;29:1039-49.

19. Kellgren JH, Lawrence JS. Radiological assessment of osteo-arthrosis. Ann Rheum Dis 1957; 16:494-502.

20. Pham T, van der Heijde D, Altman RD, et al. OMERACT-OARSI initiative: Osteoarthritis Research Society International set of responder criteria for osteoarthritis clinical trials revisited. Osteoarthritis Cartilage 2004;12:389-99.

21. Arrich J, Piribauer F, Mad P, et al. Intra-articular hyaluronic acid for the treatment of osteoarthritis of the knee: systematic review and meta-analysis. Can Med Assoc J 2005;172:1039-43.

22. Bannuru RR, Natov NS, Obadan IE, et al. Therapeutic trajectory of hyaluronic acid versus corticosteroids in the treatment of knee osteoarthritis: a systematic review and meta-analysis. Arthritis Rheum 2009;61:1704-11.

23. Bellamy N, Campbell J, Welch V, et al. Viscosupplementation for the treatment of osteoarthritis of the knee. Cochrane Database Syst Rev 2006;2:CD005321.

24. Lo GH, LaValley M, McAlindon T, et al. Intra-articular hyaluronic acid in treatment of knee osteoarthritis: a meta-analysis. JAMA 2003;290:3115-21.

25. Modawal A, Ferrer M, Choi HK, et al. Hyaluronic acid injections relieve knee pain. J Fam Pract 2005;54:758-67.

26. Wang CT, Lin J, Chang CJ, et al. Therapeutic effects of hyaluronic acid on osteoarthritis of the knee. A meta-analysis of randomized controlled trials. J Bone Joint Surg Am 2004;86-A:538-45

27. Jørgensen A, Stengaard-Pedersen K, Simonsen 0, et al. Intra-articular hyaluronan is without clinical effect in knee osteoarthritis: a multicentre, randomised, placebocontrolled, double-blind study of 337 patients followed for 1 year. Ann Rheum Dis 2010;69:1097-102.

28. Band PA. OARSI update on the evidence for osteoarthritis therapies: comment on the nomenclature used for intra-articular hyaluronan. Osteoarthritis Cartilage 2010;18:1235; author reply 1236 .

29. Altman RD, Moskowitz R. Intraarticular sodium hyaluronate (Hyalgan) in the treatment of patients with osteoarthritis of the knee: a randomized clinical trial. Hyalgan Study Group. J Rheumatol 1998;25:2203-12.

30. Clegg D0, Reda DJ, Harris CL, et al. Glucosamine, chondroitin sulfate, and the two in combination for painful knee osteoarthritis. N Engl J Med 2006:354:795-808.

31. Smith MD, Wetherall M, Darby T, et al. A randomized placebo-controlled trial of arthroscopic lavage versus lavage plus intra-articular corticosteroids in the management of symptomatic osteoarthritis of the knee. Rheumatology (Oxford) 2003:42:1477-85.

32. Zhang W, Robertson J, Jones AC, et al. The placebo effect and its determinants in osteoarthritis: meta-analysis of randomised controlled trials. Ann Rheum Dis 2008;67:1716-23.

33. Bellamy N, Campbell J, Robinson V, et al. Intraarticular corticosteroid for treatment of osteoarthritis of the knee. Cochrane Database Syst Rev 2006;2:CD005328. 